\title{
Sinonasal Organized Hematoma. Cases Report
}

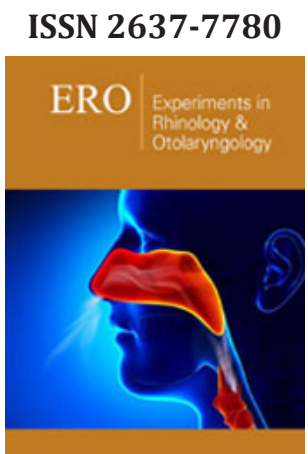

*Corresponding author: Carlos Santiago Ruggeri, Department of Otolaryngology, Chief of Rhinology Division, Hospital Italiano De Buenos Aires, Argentina

Submission: 侮 June 18, 2019

Published: 漹June 27, 2019

Volume 3 - Issue 1

How to cite this article: Rugger C, Molachino R, Legris A, Cajelli L. Sinonasal Organized Hematoma. Cases Report. Exp Rhinol Otolaryngol 3(1). ERO.000554.2019.

DOI: 10.31031/ERO.2019.03.000554

Copyright@ Carlos Santiago Ruggeri, This article is distributed under the terms of the Creative Commons Attribu $\mathrm{t}$ i o $\mathrm{n}$ 4.0 International License, which permits unrestricted use and redistribution provided that the original author and source are credited.

\author{
Ruggeri CS*, Molachino R, Legris A and Cajelli L
}

Department of Otolaryngology, Argentina

\section{Abstract}

The organized hematoma of paranasal diseases is a rare disease, caused by hemorrhage, fibrosis and neovascularization. It most frequently involves the maxillary sinus and has clinical and imaging symptoms and signs similar to other benign or malignant paranasal sinus tumors. Its location in the sphenoid sinus is very infrequent, 6 cases have been described in the literature and in two reported as permanent sequels alterations in vision. Two clinical cases of hematomas organized in the maxillary sinus and in the sphenoid are described in this study and a review of the literature on this infrequent disease was made.

Keywords: Organized hematoma; Paranasal sinuses; Endoscopic sinus surgery

\section{Introduction}

Organized sinus hematoma is an infrequent disease, caused by hemorrhage, fibrosis and neovascularization. Due to the expansive characteristics and local destruction that can occur, it can be difficult to make the differential diagnosis with other benign or malignant tumors of the paranasal sinuses. The treatment is surgery and the endonasal approach with endoscopes is the most used technique to resect these pseudo tumors. In this study we describe the cases of two patients who had organized hematomas in the maxillary and sphenoid sinuses. A review of the literature on this rare disease was made.

\section{Clinical Cases}

\section{Case 1}

Male patient of 67 years of age. He consulted for intermittent epistaxis of the right nasal fossa of 3 months of evolution. He had a history of anticoagulant treatment. By nasal endoscopy, hematic remnants were observed, and the sinus computed tomography showed an occupation of the right maxillary sinus with bone destruction of the intersinusonasal wall (Figure 1). An endonasal approach was made with endoscopes and an ungiectomy, right maxillary antrostomy and resection of friable consistency polypoid tissue were performed. The deferred anatomopathological study reported: "Extensive areas with necrosis, hemorrhage and fibrin leukocytic exudate" compatible with an abscessed polyp associated with an organized hematoma. The patient was discharged 24 hours after the intervention and remained asymptomatic and without evidence of tumor in the endoscopic controls performed for 3 years.
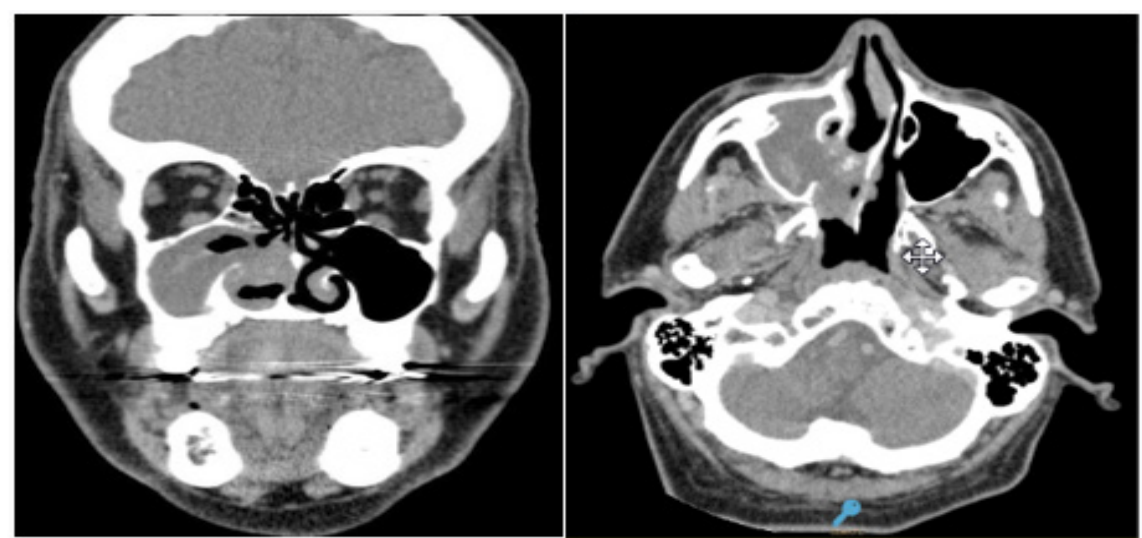

Figure 1: CT Organized hematoma of the maxillary sinus with destruction of the intersinusonasal wall. 


\section{Case 2}

A 49-year-old man with a history of combined endoscopy-assisted cranionasal approach and postoperative chemoradiotherapy seven years earlier, for an esthesioneuroblastoma that involved ethmoids and had an endocranial extension. During routine checks, and for referring intermittent headache, imaging studies were requested. Computed tomography and nuclear magnetic resonance with contrast showed two lesions of different density compatible with sphenoid mucocele. Through an endonasal approach with endoscopes and intraoperative navigation, a wide sphenoidotomy was performed and the mucocele was marsupialized, aspirating mucous secretions. A red and round, non-pulsatile tumor was observed behind, in contact with the roof and the left wall of the sphenoid sinus. The tumor was resected with cutting forceps. He was discharged 24 hours after the surgery. The deferred pathological study reported "Hematic material with fibrin, some lymphocytes and leukocytes" compatible with sphenoid organized hematoma. The patient had no more headache and in the endoscopic controls for 2 years the sphenoidal sinus was ventilated and without recurrence of the hematoma (Figure 2).
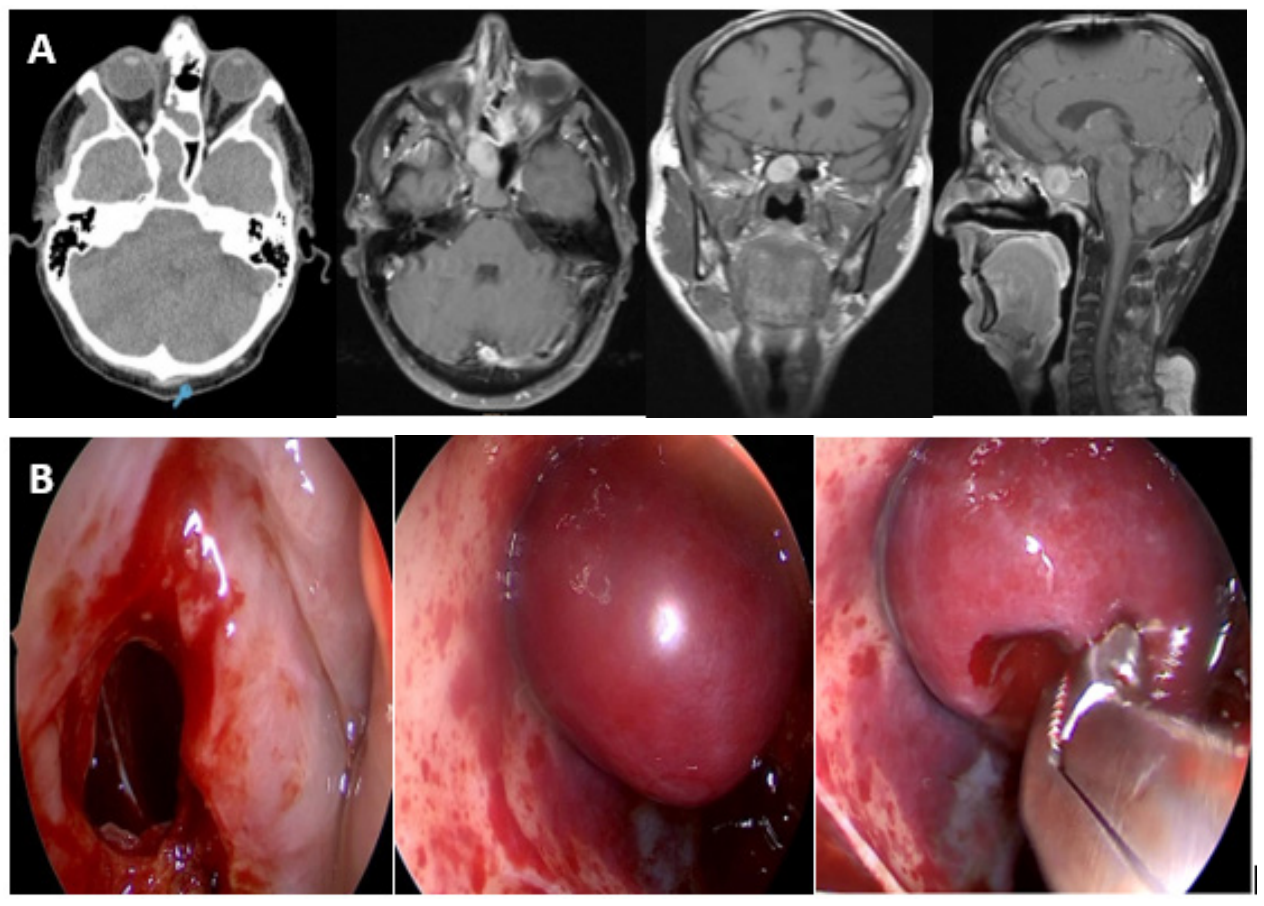

Figure 2: A: Organized hematoma of the sphenoid sinus-tomography and magnetic resonance with contrast.

B: Transnasal sphenoidotomy and resection of an organized hematoma.

\section{Discussion}

Organized hematoma of the paranasal sinuses is a rare pathology that causes sinus inflammation and bone destruction. The etiology is unknown but is believed to be initiated by the accumulation of blood in the paranasal cavities by various factors. The inadequate ventilation and drainage of the affected sinus, accompanied by the formation of a fibrous capsule, prevents the resorption of the hematoma and results in neovascularization and fibrosis with recurrent intracapsular bleeding and expansive growth. Depending on their histological characteristics, they can be classified as edematous, glandular, fibrous, cystic and angiomatous. It represents between 4 to $5 \%$ of nasosinusal polyps. Its location in the maxillary sinus is more frequent and patients may have a history of pathologies that can produce epistaxis. In a retrospective study they described 84 patients with organized hematomas, 82 involved the maxillary sinus and two the nasal cavity.

Thirty-nine were men and 45 women, and the average age was 50.2 years. The most frequent symptoms were epistaxis $(60 / 84-$
$71.4 \%$ ) and nasal obstruction (47/84-60\%), followed by pain in the cheek $(n=11)$, headache $(n=7)$, epiphora $(n=5)$ and exophthalmos $(n=2)$. Twenty-five patients (30\%) had a history of rhinosinusal surgery and 12 of antiaggregant treatment with aspirin, 7\% had liver cirrhosis and 5\% had renal failure. All antecedents that can produce epistaxis. Seventy percent had bone destruction of one of the walls of the maxillary sinus [1]. In other studies, they also described the location of the hematoma in the maxillary sinus as more frequent [2-4].

All were treated with surgery, using an endonasal approach with endoscopes. Our first case coincides with the literature in which the patient had a history of a treatment that favored bleeding (anticoagulants). It was successfully operated by an endonasal approach with endoscopes. The localization of organized hematomas in other paranasal cavities is very rare [5-7].

A systematic search was made in the bibliography using the key words "Shenoid sinus organized hematoma" and [6] cases of hematomas located in the sphenoid sinus were found in PUBMED. 
Wu and collaborators [3] reported a lesion located in the sphenoid sinus among seven patients described with organized sinus hematomas and in other studies they described one located in the sphenoid and one in the frontal sinus [2], and among seventeen patients they found only one in the sphenoid and another in the frontal [4]. In three other studies they described isolated clinical cases of organized hematomas in the sphenoid sinus. One caused destruction of the sella turcica and simulated a pituitary tumor, was operated by a transnasal transsphenoidal approach with endoscopes [5] and the other caused a decrease in visual acuity with epistaxis, and retroorbital headache, and despite endonasal decompressive surgery with endoscopes the patient was left with a decrease in visual acuity.

In the third case, they described a patient with a sphenoid hematoma that caused decreased visual acuity and palpebral ptosis, with mydriasis and atrophy of the second cranial nerve that did not improve his vision after surgery [7]. This highlights the importance of knowing this pathology and that although it is histologically benign it must be treated early because of its potential to generate serious complications due to the involvement of adjacent vital structures. In our clinical case 2 , the patient had few symptoms but when performing the sphenoidotomy the hematoma was found in relation to the lateral wall of the sphenoid and in relation to the II pair and the internal carotid artery. It is important to know the existence of this infrequent pathology due to its clinical and imaging similarity with other benign and malignant tumors of the paranasal sinuses. The exegesis of the organized hematoma must be complete and since the lesion is easy to resect, the endonasal video-endoscopic approach is preferred because of the lower morbidity produced by the surgery.

\section{Conclusion}

The organized sinus hematoma is a benign and infrequent pathology. It is important to know its existence in order to make the differential diagnosis with other benign and malignant tumors of the paranasal sinuses. The involvement of the sphenoid sinus is exceptional and may be associated with serious complications due to the injury of the adjacent anatomical structures. The treatment is surgical and the endonasal technique with endoscopes is of choice due to its effectiveness and low morbidity.

\section{References}

1. Pang W, Hu L, Wang H, Sha Y, Ma N, et al. (2016) Organized hematoma: an analysis of 84 cases with emphasis on difficult prediction and favorable management. Otolaryngol Head Neck Surg 154(4): 626-633.

2. Kim JS, Seok Oh J, Kwon SH (2016) The increasing incidence of paranasal organizing hematoma: a 20 -year experience of 23 cases at a single center. Rhinology 54(2): 176-182.

3. Wu AW, Ting JY, Borgie RC, Busaba NY, Sadow PM, et al. (2013) Diagnostic characteristics of sinonasal organizing hematomas: avoiding misdiagnosis. Int Forum Allergy Rhinol 3(7): 598-602.

4. Choi SJ, Seo ST, Rha KS, Kim YM (2015) Sinonasal organized hematoma: clinical features of seventeen cases and a systematic review. Laryngoscope 125(9): 2027-2033.

5. Nakagawa T, Kawai Y, Sakamoto T, Ito J (2010) Organized haematoma of the sphenoid sinus mimicking a pituitary tumour. J Laryngol Otol 124(1): 83-85.

6. Yoon YH, Kim SH, Kim YM, Rha KS (2018) Organized hematoma of the sphenoid sinus causing acute visual loss. Eur J Ophthalmol 28(6): NP7NP9.

7. Lin YH, Wang PC, Lin YS (2016) Sphenoid sinus organized hematoma with cranial neuropathies masquerading as a malignancy: A Case Report. Oncol Lett 11(6): 3571-3574. 